minimum (no thermal corrections) and maximum thermal response models of the probe ultrastable oscillator. Given our current understanding of the high-temperature response of this oscillator, it is now seen that the tendency reported previously ${ }^{1}$ towards increasing winds in deepest regions explored by the probe-has disappeared.

The above-mentioned wind measurements by the Atmospheric Structure Instrument's accelerometers ${ }^{8}$ are particularly reassuring in that they are obtained by an entirely different technique that does not depend on radio tracking, the stability of a crystal oscillator, or the substraction of other contributing velocities. They are of low velocity resolution and limited accuracy, however, and therefore do not give precise wind speeds. The radio tracking measurements from Earth (by the Very Large Array) have a much better viewing angle than that available from the overhead Galileo orbiter, but were significantly affected by the unknown frequency offset of the probe ultrastable oscillator and the enormous transmission distance leaving only a faint signal at Earth which must be extracted from the noise ${ }^{9}$. It is remarkable that these three techniques agree on the essential description of the winds, leaving little doubt that they have been characterized.

Although there was speculation about whether the winds were shallow or deep, very few had predicted that the winds would increase with depth. By 'shallow' one usually means 'above the base of the water cloud', that is, above the 5-bar level. This is the region where sunlight is absorbed and latent heat is released, so it was assumed that if the winds drew their energy from these sources they should decay with depth below the cloud base $\mathrm{e}^{2,10-12}$. On the other hand, if the winds drew their energy from internal heat, they might continue down indefinitely into the fluid interior of Jupiter ${ }^{13-15}$. Only one analysis ${ }^{16}$ predicted that the winds would increase with depth. The DWE has shown that the winds are 'deep'. But it is not clear that this means that they are powered by internal heat; neither is it clear why the winds increase with depth at the 1-4 bar level.

In the atmospheres of rotating planets, the variation of zonal wind with depth is proportional to the variation of density with latitude. The density gradient is taken at constant pressure. If the gradient is zero, the fluid is said to be barotropic. If the interior of a fluid planet is barotropic, then each cylinder, concentric with the axis of rotation, will rotate as a solid body. These statements, proved nearly a century ago ${ }^{17}$, are valid when forcing and dissipation are small. The forcing that maintains the winds may be confined to the surface layers or it may be distributed throughout the fluid. Thus we cannot say with certainty that the zonal winds are powered by internal heat.

The fluid is clearly not barotropic in the 1-4 bar pressure range where zonal wind is increasing with depth. The increase implies a density decrease of $0.5 \%$ per degree of latitude at the $1-2$ bar levels. This is three to four times larger than, but of the same sign as, that inferred from the temperature gradients observed at the 150- and 270 -mbar levels ${ }^{18}$. The latter have been interpreted as a sign of upwelling to the south, and downwelling to the north, of the probe site. The DWE results suggest that the upwellings and downwellings extend at least to the 4-bar level.

The constancy of the wind in the 4-21 bar range implies that the fluid there is barotropic. This is expected, as sunlight does not penetrate $^{19}$ and latent heat is not released at these levels. Convection and radiation, the other modes of heat transfer, are likely to maintain a barotropic state $e^{20}$. Thus the $170 \mathrm{~m} \mathrm{~s}^{-1}$ winds measured by the DWE probably extend well into the fluid interior of Jupiter. This result is qualitatively consistent with studies of cloud-top winds, whose vorticity can be used to infer the winds at depth ${ }^{21}$. Magnetic fields ${ }^{22}$, phase transitions, and small gradients of temperature can alter the barotropic state, so the zonal winds do not necessarily extend throughout the planet. But the DWE result shows that the winds are deep, and this has important implications for atmospheric dynamics.
Received 7 March; accepted 3 June 1997.

1. Atkinson, D. H., Pollack, J. B. \& Seiff, A. Galileo Doppler measurements of the deep zonal winds at Jupiter. Science 272, 842-843 (1996).

2. Pollack, J. B., Atkinson, D. H., Seiff, A. \& Anderson, J. D. Retrieval of a wind profile from the Galileo probe telemetry signal. Space Sci. Rev. 60, 143-178 (1992).

3. Limaye, S. S. Jupiter: new estimates of the mean zonal flow at the cloud level. Icarus 65, 335-352 (1986)

4. Seiff, A. et al. Structure of the atmosphere of Jupiter: Galileo probe measurements. Science 272, 844845 (1996).

Ingersoll, A. P. et al. Interaction of eddies and mean zonal flow on Jupiter as inferred from Voyager 1 and 2 images. J. Geophys. Res. 86, 8733-8743 (1981).

6. Orton, G. et al. Earth-based observations of the Galileo probe entry site. Science 272, 839-840 (1996).

Beebe, R. F., Simon, A. A. \& Huber, L. F. Comparison of Galileo probe and Earth-based translation rates of Jupiter's equatorial clouds. Science 272, 841 (1996).

8. Seiff, A. et al. Wind speeds measured in the deep jovian atmosphere by the Galileo probe accelerometers. Nature 388, 650-652 (1997).

9. Folkner, W. M. et al. Earth-based radio tracking of the Galileo probe for Jupiter wind estimation. Science 275, 644-646 (1997).

10. Hess, S. L. \& Panofsky, H. A. in Compendium of Meteorology (ed. Malone, T. F.) 391-398 (Am. Meteorol. Soc., Boston, 1951).

11. Ingersoll, A. P. \& Cuzzi, J. N. Dynamics of Jupiter's cloud bands. J. Atmos. Sci. 26, 981-985 (1969).

12. Williams, G. P. Jovian dynamics. Part I: vortex stability, structure, and genesis. J. Atmos. Sci. 53, 2685 2734 (1996).

13. Ingersoll, A. P. \& Cuong, P.-G. Numerical model of long-lived jovian vortices. J. Atmos. Sci. 38, 2067 2076 (1981).

14. Busse, F. H. A simple model of convection in the jovian atmosphere. Icarus 29, 255-260 (1976)

15. Ingersoll, A. P. \& Pollard, D. Motion in the interiors and atmospheres of Jupiter and Saturn: scale analysis, anelastic equations, barotropic stability criterion. J. Atmos. Sci. 52, 62-80 (1982).

16. Dowling, T. E. Estimate of Jupiter's deep zonal-wind profile from Shoemaker-Levy 9 data and Arnol'd's second stability criterion. Icarus 117, 439-442 (1995).

17. Poincaré, H. Sur la Précession des corps déformables. Bull. Astron. 27, 231-356 (1910).

18. Gierasch, P. J., Conrath, B. J. \& Magalhaes, J. O. Zonal mean properties of Jupiter's upper troposphere from Voyager infrared observations. Icarus 67, 456-483 (1986).

19. Sromovsky, L. A. et al. Solar and thermal radiation in Jupiter's atmosphere: initial results of the Galileo probe net flux radiometer. Science 272, 851-854 (1996).

20. Ingersoll, A. P. \& Porco, C. C. Solar heating and internal heat flow on Jupiter. Icarus 35, $27-43$ (1978). 21. Dowling, T. E. \& Ingersoll, A. P. Jupiter's great red spot as a shallow water system. J. Atmos. Sci. 46, 3256-3278 (1989)

22. Kirk, R. L. \& Stevenson, D. J. Hydromagnetic constraints on deep zonal flow in the giant planets. Astrophys. J. 316, 836-846 (1987).

Acknowledgements. We thank the Galileo Project and NASA's Planetary Atmospheres Program. This work was supported by NASA.

Correspondence and requests for materials should be addressed to D.H.A. (e-mail: atkinson@maxwell. ee.uidaho.edu).

\section{Wind speeds measured in the deep jovian atmosphere by the Galileo probe accelerometers}

A. Seiff ${ }^{\dagger}$, R. C. Blanchard $\ddagger$, T. C. D. Knight $\$$, G. Schubert $\|$, D. B. Kirk $₫$, D. Atkinsong, J. D. Mihalov ${ }^{\star} \&$ R. E. Young ${ }^{\star}$

*NASA-Ames Research Center, Moffett Field, California 94035, USA

$\dagger$ Department of Meteorology, San Jose State University, San Jose, California, USA

†ASA Langley Field, MS-107, Hampton, Virginia 23665, USA

$\|$ Department of Earth \& Space Science, UCLA, Los Angeles, California 90024, USA

I Department of Electrical Engineering, University of Idaho, Moscow,

Idaho 83844, USA

The atmosphere of Jupiter has a complex circulation which, until recently, has been observable only at the cloud tops ${ }^{1,2}$; the mechanisms driving the winds, and the nature of the interior circulation, remained unknown ${ }^{3}$. Recent analyses ${ }^{4-6}$ of the radio signal from the Galileo probe, obtained during its descent into the jovian atmosphere, have suggested a vigorous interior circulation below the 4-bar level. Here we report an independent measurement of the winds below the cloud tops, making use of the data obtained by the two accelerometers on the descending probe. We find evidence for two distinct wind regimes, in general agreement with the Doppler radio measurements: a region of wind shear between 1 and 4 bar, where the wind speed increases dramatically with depth; and then a region of constant high-velocity winds down to at least the 17-bar level.

We present here acceleration data and their interpretation to 
define zonal wind velocities. Two identical sensors, $z_{1}$ and $z_{2}$, aligned with the probe axis of symmetry (the $z$ axis) returned data shown in Fig. 1 during 59 minutes of probe descent on the parachute. The data are plotted as functions of atmospheric pressures measured by the Atmosphere Structure Instrument ${ }^{7,8}$. In a frame of reference moving with the wind, the $z$ axis and the descent velocity were close to vertical during parachute descent. As expected, the measured accelerations agree approximately with Jupiter's acceleration due to gravity, $g$. Measured accelerations were initially greater than $g$, then fell below $g$ at atmospheric pressure $p>2$ bar. For $p>4$ bar, the data are roughly parallel to the $g$ curve. Data from the two sensors are generally quite similar.

Deviations of the measured accelerations, $a_{z}$, from the acceleration due to gravity are explained by inputs other than gravity, of which there were two, as represented in the following equation:

$$
a_{z}=a_{\mathrm{g}}-\omega^{2} R \cos ^{2} \theta+\mathrm{d} w / \mathrm{d} t+L(\pi \delta / T)^{2}
$$

Here, following the convention used in geodesy, $a_{\mathrm{g}}$ is the gravitational acceleration (uncorrected for centrifugal acceleration), $\omega$ is the planetary rotational velocity $\left(\mathrm{rad} \mathrm{s}^{-1}\right), R$ is the probe radial distance from the centre of the planet, and $\theta$ is the joivicentric latitude. Thus $\omega^{2} R \cos ^{2} \theta$ is the radial component of centrifugal acceleration due to planetary rotation. Together, the first two terms comprise $g$, the gravity acceleration. Variation of the gravitational acceleration $a_{\mathrm{g}}$ with radius was represented simply by $a_{\mathrm{g}}=a_{\mathrm{g} 0}\left(R_{0} / R\right)^{2}$ where subscript 0 identifies the 1-bar level. The two additional terms are: first, $\mathrm{d} w / \mathrm{d} t$, the time rate of change of descent velocity, which is the real deceleration of the probe resulting from increasing atmospheric density; and second, $L(\pi \delta / T)^{2}$, the mean acceleration over one oscillation cycle of the probe swinging as a pendulum beneath the parachute (here $L$ is the pendulum length, $\delta$ is the swinging amplitude and $T$ is the period). Both terms add to tension in the parachute cord, and increase the acceleration felt by the probe. Analogously, the restraining force needed to hold the accelerometer test mass in fixed position, which is the measure of the acceleration, is increased. The observed swinging motion executed $\sim 3$ cycles in $16 \mathrm{~s}$ with $L=13.9 \mathrm{~m}, \delta=2.2^{\circ}$ and $T=4.9 \mathrm{~s}$ (ref. 8): this defines a mean pendulum acceleration of $0.0084 \mathrm{~m} \mathrm{~s}^{-2}$. The real deceleration of the probe, $\mathrm{d} w / \mathrm{d} t$, was derived by differentiating the descent velocities, shown in Fig. 2. It is evident that this term explains the excess of measured acceleration over $g$ early in the descent (Fig. 1).

From equation (1), we expect $a_{z}-\mathrm{d} w / \mathrm{d} t-L(\pi \delta / T)^{2}$ to be a measure of $g$. However, the data so adjusted do not agree with $g$, unless allowance is made for additional centrifugal acceleration associated with winds (Fig. 3). With zonal winds present, $\omega=$ $\omega_{\mathrm{j}}+u_{\mathrm{w}} / R \cos \theta$ where $\omega_{\mathrm{j}}$ is Jupiter's rotational velocity and $u_{\mathrm{w}}$ is wind velocity, and two new terms appear on the right-hand side of equation (1), $2 \omega_{\mathrm{j}} u_{\mathrm{w}} \cos \theta+u_{\mathrm{w}}^{2} / R$. Term magnitudes at $u_{\mathrm{w}}=100 \mathrm{~m} \mathrm{~s}^{-1}$ are $2 \omega u_{\mathrm{w}} \cos \theta=0.03495 \mathrm{~m} \mathrm{~s}^{-2}$ and $u_{\mathrm{w}}^{2} / R=0.00014 \mathrm{~m} \mathrm{~s}^{-2}$. Curves of $g$ corrected for these terms are shown in Fig. 3 for wind velocities of 100,200 and $300 \mathrm{~m} \mathrm{~s}^{-1}$.

In relation to these curves, the data indicate the presence and the magnitude of the winds. They clearly indicate a shear layer between $\sim 1.5$ and 4 bar terminating in high velocities $\left(\sim 200 \mathrm{~m} \mathrm{~s}^{-1}\right)$ which then persist below the 4-bar level for the remainder of the descent. The two main characteristics of the Doppler winds, the existence of a shear layer from 1.5 to 4 bar and essentially constant deep atmosphere winds, are thus confirmed. This is an important result as it gives information about the planet's internal circulation.

The wind magnitude definition by the accelerometers is limited by the relatively coarse digital resolution of the measurement, with 1 count equivalent to $87.4 \mathrm{~m} \mathrm{~s}^{-1}$ of zonal velocity. A 1-count uncertainty is rather typical of digital data. Data taken during cruise

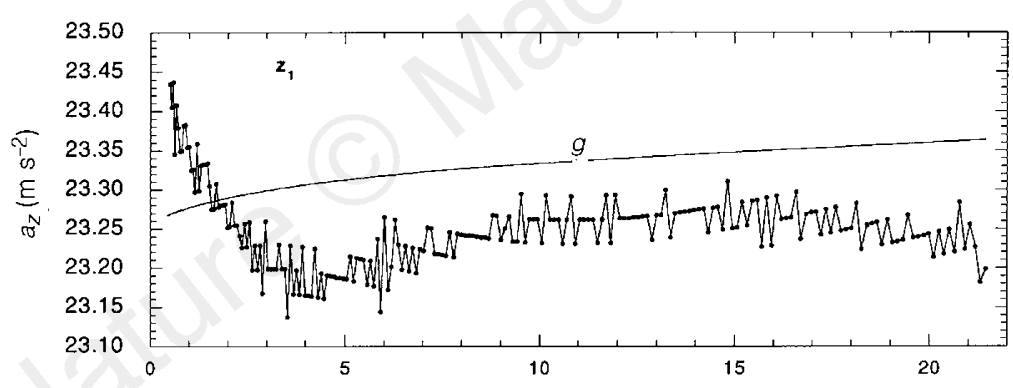

Figure 1 Axial accelerations $a_{z}$ of the Galileo probe (data points) measured during parachute descent ( $p$ is atmospheric pressure). Data are shown from accelerometers $z_{1}$ (top panel) and $z_{2}$ (bottom panel). Each value plotted is the average of 512 readings in a 16-s sampling interval. The scatter is \pm 1 count about the local mean, with 1 count $=0.031 \mathrm{~m} \mathrm{~s}^{-2}$. The gravity curve $g$, which is corrected for planetary spin, varies perceptibly with radial distance to the planet's centre, and hence with pressure. For the measured gravitational field ${ }^{10}$ and $\omega_{\mathrm{j}}=0.00017585 \mathrm{rad} \mathrm{s}^{-1}$, the gravitational and centrifugal accelerations at the 1 -bar level at the entry site are 25.456 and $2.178 \mathrm{~m} \mathrm{~s}^{-2}$, respectively, giving $a_{g}=a_{g 0}=23.278 \mathrm{~ms}^{-2}$. At the probe descent latitude, $\theta=6.42^{\circ}, R_{0}=71,334 \mathrm{~km}$ at the 1 -bar level, corresponding to an equatorial radius of $71,400 \mathrm{~km}$ (ref. 10).
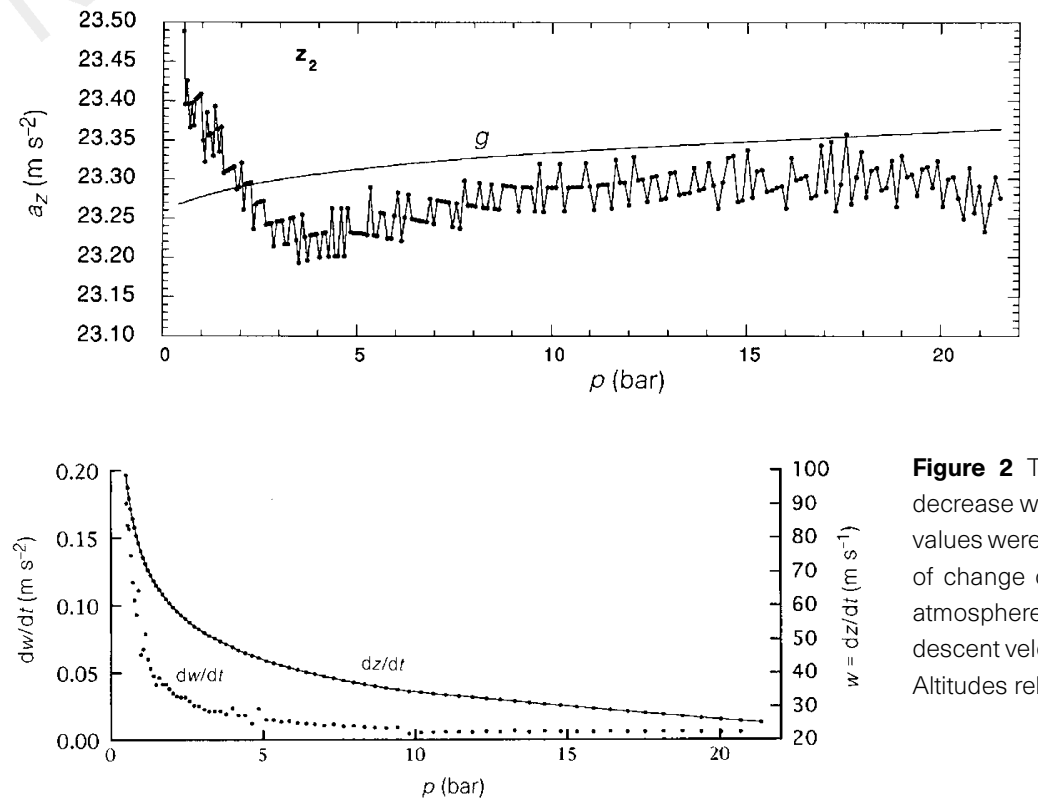

Figure 2 The descent velocity $(\mathrm{d} z / \mathrm{d} t)$ and the probe deceleration $(\mathrm{d} w / \mathrm{d} t)$ decrease with increasing atmospheric pressure $(p)$ in parachute descent. These values were derived, with the assumption of hydrostatic equilibrium, from the rate of change of pressure with time indicated by pressure data from the Galileo atmosphere structure experiment. These data also define the contribution of descent velocity to line-of-sight velocity required in the Doppler wind experiment. Altitudes relative to the 1 -bar level were from +16 to $-129 \mathrm{~km}$. 


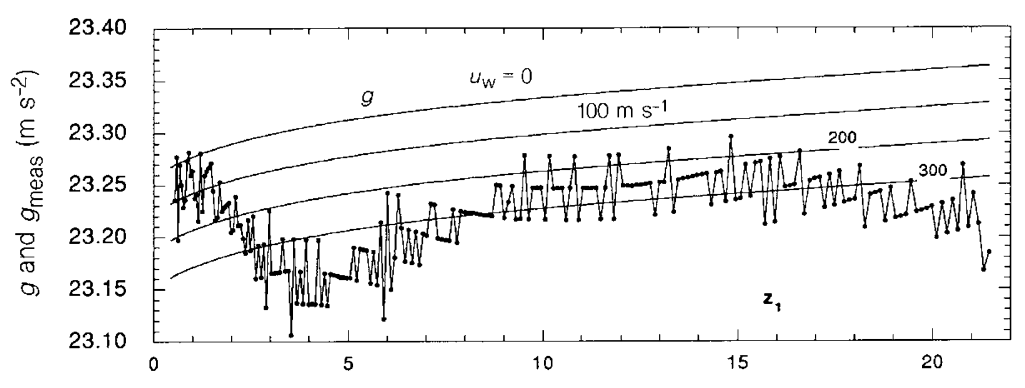

Figure 3 Data from accelerometers $z_{1}$ (top panel) and $z_{2}$ (bottom panel), reduced by the real deceleration of the probe $(\mathrm{d} w / \mathrm{d} t)$ and probe swinging accelerations, compared with gravity accelerations modified for several zonal wind speeds $u_{\mathrm{w}}$. The comparisons indicate wind speeds increasing between the 0.5 - and the 4-bar levels, then becoming constant at $\sim 200 \mathrm{~m} \mathrm{~s}^{-1}$. In each panel, the uppermost $g$ curve is for zero wind. The three lower curves indicate accelerations of gravity experienced by a probe moving with the winds at 100,200 and $300 \mathrm{~m} \mathrm{~s}^{-1}$.

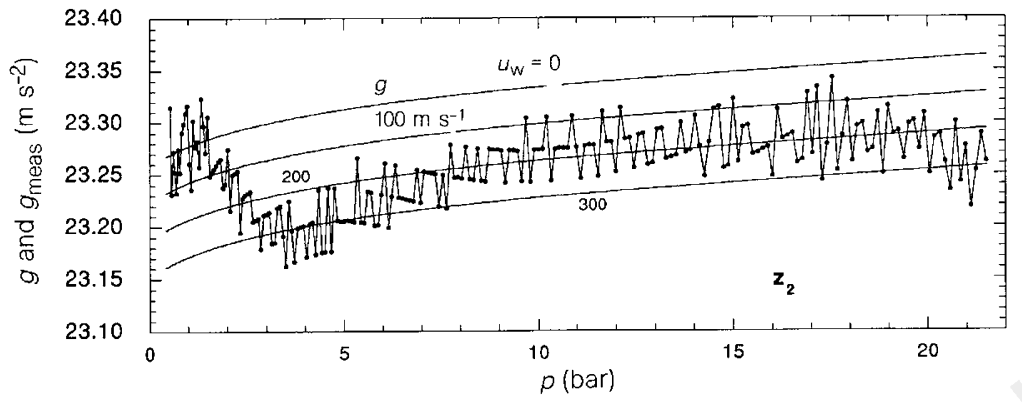

checks and on the ground indicate that system offsets (sensors plus electronics) were stable within $\sim 1$ count. The sensor offsets were shown-by analogue calibrations in 1983-85-to be stable within 0.15 counts $\left(z_{1}\right)$ and 0.05 counts $\left(z_{2}\right)$ over a period of 18 months. Self checks of the electronics during descent and the data themselves indicate that the offset was stable within $\sim 1$ count during the descent. There were, however, two disturbing influences: just before descent, the probe experienced the $228 \mathrm{~g}$ deceleration of entry. Immediately thereafter, they were exposed to a probe internal environmental temperature swing from 0 to $-35^{\circ} \mathrm{C}$ and finally to $+115^{\circ} \mathrm{C}$. The sensors were designed and calibrated for the operating range from -20 to $+50^{\circ} \mathrm{C}$. Outside this range, it was necessary to extrapolate the calibrations. Below 2 bar and from 9 to 17 bar data were taken at sensor temperatures within the calibration range.

To correct for the large and rapid variation in probe internal temperature, offset or zero reading shifts were calculated, based on data taken in 1983 and 1984 during calibration temperature sweeps at rates of $\sim 1{ }^{\circ} \mathrm{C} \mathrm{min}{ }^{-1}$. The $\mathrm{z}_{2}$ correction was 1.6 counts after passing through the temperature minimum while the $\mathrm{z}_{1}$ correction was a maximum of 0.3 counts. The corrected data indicate wind speeds of $230 \pm 30 \mathrm{~m} \mathrm{~s}^{-1}\left(\mathrm{z}_{1}\right)$ from 9 to 16 bar, and $\sim 180 \mathrm{~m} \mathrm{~s}^{-1}\left(\mathrm{z}_{2}\right)$ between 8 and 18 bar. The $z_{2}$ data parallel the $g$ contour, implying constant wind speed. The Doppler wind magnitude in the deep atmosphere, $175 \mathrm{~m} \mathrm{~s}^{-1}$ with formal uncertainty $\sim \pm 5 \mathrm{~m} \mathrm{~s}^{-1}$, also agrees with the $z_{2}$ accelerometer determination. The disagreement of the two sensors is within the 1-count uncertainty.

From 0.6 to 1.5 bar, the present data indicate relatively constant winds at speeds somewhat smaller than the cloud-top winds measured from Voyager images $\left(90-150 \mathrm{~m} \mathrm{~s}^{-1}\right)^{1,2}$. The $\mathrm{z}_{1}$ data indicate velocities generally $<100 \mathrm{~m} \mathrm{~s}^{-1}$ whereas the $\mathrm{z}_{2}$ data indicate near zero velocity on average. (We feel that the sensor zero reading is in question there.) These data are, however, within 1 count of agreeing with previously observed cloud-top winds.

The general agreement between the determinations of the winds by the accelerometers and by Doppler tracking is highly significant. Both measurements indicate two distinct wind regimes: the region of wind shear with winds increasing from 1 to 4 bar; and the region of constant, high-velocity winds $\left(\sim 200 \mathrm{~m} \mathrm{~s}^{-1}\right)$ from 4 to at least 17 bar. Below the 4-bar level, the measured winds are probably driven by deep-seated processes associated with the outflow of internal heat, whereas above the 4-bar level, 'surface' effects modify the deep winds. To modify the winds vertically, meriodional temperature gradients are needed. ${ }^{5}$ These can be provided by the meridional differences in cloud cover, of which the belts and zones are the most prominent ${ }^{9}$ : these cloud cover variations are accompanied by differences in solar heating and heat of condensation, and so are a source of meridional temperature contrast.

Received 10 March; accepted 25 June 1997.

1. Limaye, S. et al. Jovian winds from Voyager 2, Part I: zonal mean circulation. J. Atmos. Sci. 39, 14131432 (1982).

2. Beebe, R. F., Simon, A. A. \& Huber, L. F. Comparison of Galileo Probe and Earth-based translation rates of Jupiter's equatorial clouds. Science 272, 841 (1996).

3. Pollack, J. B., Atkinson, D. H., Seiff, A. \& Anderson, J. D. Retrieval of a wind profile from the Galileo Probe telemetry signal. Space Sci. Rev. 60, 143-178 (1992).

4. Atkinson, D. P., Pollack, J. B. \& Seiff, A. Galileo Doppler measurements of the deep zonal winds at Jupiter. Science 272, 842-843 (1996).

5. Atkinson, D. H., Ingersoll, A. P. \& Seiff, A. Deep winds on Jupiter as measured by the Galileo probe. Nature 388, 649-650 (1997).

6. Folkner, W. M. Earth-based radio tracking of the Galileo Probe for Jupiter wind estimation. Science 275, 844-845 (1997).

7. Seiff, A. \& Knight, T. C. D. K. The Galileo Probe atmosphere structure instrument. Space Sci. Rev. 60, 203-232 (1992).

8. Seiff, A. et al. Structure of the atmosphere of Jupiter: Galileo Probe measurements. Science 272, 844845 (1996).

9. Ingersoll, A. P. \& Cuzzi, J. N. Dynamics of Jupiter's cloud bands. J. Atmos. Sci. 26, 981-985 (1969). 10. Anderson, J. D. in Jupiter (ed. Gehrels, J.) 113-121 (Univ. Arizona Press, Tucson, 1976).

Correspondence should be addressed to A.S. at MS 245-1, NASA-Ames Research Center, Moffett Field, California 94035, USA (e-mail: aseiff@mail.arc.nasa.gov).

\section{Spontaneous polarization in dense hydrogen}

\section{B. Edwards \& N. W. Ashcroft}

Laboratory of Atomic and Solid State Physics, Cornell University, Ithaca, New York 14853-2501, USA

More than six decades have passed since Wigner and Huntington ${ }^{1}$ proposed that hydrogen might form a solid metallic phase at high density with characteristics similar to the alkali metals. This possibility has been investigated using the diamond-anvil cell to compress the crystalline state of molecular hydrogen ${ }^{2}$, but there is still no definitive evidence for a dense, low-temperature metallic state. Below $140 \mathrm{~K}$, solid hydrogen undergoes a transition at about 1.5 million atmospheres between two orientationally ordered states. The intermolecular vibrational mode (the vibron) shifts to a lower frequency at this transition ${ }^{3,4}$, and becomes strongly infrared-active ${ }^{5}$. So far as is known, hydrogen remains in this phase to the highest pressures yet reached. Here we report 